\title{
Joint Coordinated Multicell Processing and MIMO: Selection of Base Stations and Gain
}

\author{
Virgile Garcia ${ }^{1}$, Nikolai Lebedev ${ }^{1,2}$ and Jean-Marie Gorce ${ }^{1}$ \\ Contacts: virgile.garcia@insa-lyon.fr, lebedev@cpe.fr, jean-marie.gorce@insa-lyon.fr \\ ${ }^{1}$ University of Lyon, INRIA \\ ${ }^{1}$ INSA-Lyon, CITI, F-69621, Villeurbanne, France \\ ${ }^{2}$ CPE Lyon, BP 2077, F-69616, France
}

\begin{abstract}
In this paper, we provide the analysis of the downlink Coordinated Multiple Point (CoMP) used in conjunction with the basic MIMO. The CoMP is the joint multi-cell transmission from several BS to mobiles, coupled here to an open-loop MIMO technique that does not require the perfect channel state knowledge. We show by simulation, that even for $4 \times 4$ MIMO transmission, the CoMP can improve the spectral eff ciency for some mobiles, depending on capacity outage requirements.
\end{abstract}

Keywords: Multi-cell processing, MIMO, capacity distribution, macro-diversity, small cells.

\section{INTRODUCTION}

Most of the upcoming wireless cellular systems standards implement a Multiple-Input Multiple-Output processing to exploit the spatial dimension, which is one of the key factors enabling high data rates over the wireless channel. More recently, a distributed MIMO in a multi-cell environment appeared as a breakthrough technology to improve the energycapacity trade-off in cellular networks [1]. On the other side, a network-wide, rather then single-link oriented vision lead to the introduction of the so-called Coordinated Multiple Points (CoMP), not for providing a huge increase of the total throughput [2], but rather to improve the SINR of cell edge users through the distributed spatial diversity. This is motivated by the obvious fact that in a very dense deployments with the reduced cell size and strong spatial frequency reuse (possibly with the whole spectrum resource made available within each cell, reuse-1 [3], [4], the interference becomes the main limiting factor, especially at cell boundaries.

The main idea behind the CoMP is to enable the users at the cell edge to decode the joint transmissions from two or more BSs in the same frequency band. That way, a better coverage is ensured with less users experiencing outage, and thus, a more intense reuse of the spectrum in dense areas is allowed. Several cooperation techniques of CoMP have been studied in [5], [6]. The maximal sum-rate capacity has been derived in [1] providing an upper-bound and revealing the potential of the distributed MIMO.

Both CoMP and MIMO are often coupled to the Orthogonal Frequency Division Multiple Access (OFDMA) which became a de facto access technology for many of current and futur standards, due to the intrinsic capability of OFDM to compensate for the fading and to offer the $\mathrm{f}$ ne granularity for resources sharing, by aggregating them into time-frequency resource blocks (RB).

The allocation of those RB either by using the interference avoidance via pattern based full or fractional reuse methods [3], [7], [8] or more recent interference coordination (ICIC) methods can be eff cient only for large scale centrally planned cellular systems, whereas the future systems for dense areas with sporadic cells deployment will necessitate the automated network planning and optimisation. This has motivated the development of the concept of Self-Optimized and Self-Organized Networks (SON) [9].

An example of a SON-compliant technique is the distributed power allocation and scheduling algorithm for the downlink (DL) based on local Signal-to-Interference Ratio (SIR) information only fed back by the mobile, proposed in [10]. In every slot, the most interfering resources are switched off as long as this contributes to the overall capacity increase. Other contributions studied the inf uence of the partial feedback [11].

The main issue with the coordination is the huge amount of signalling required to exchange channel data, algorithms related parameters, as well as synchronisation data making it impractical due to backhaul limitations. Some CoMP variants, such as distributed beamforming or dirty-paper-coding require a perfect time synchronisation in order to distributively generate in-phase signals or eff cient precoding patterns [5]. We choose to rely on a practical Alamouti-like MIMO that solely requires users' mean Signal-to-Interference and Noise Ratios (SINR), computed from powers received from BS, but not the perfect CSI with estimation of channel coeff cients. Thus, the required synchronisation between cooperating cells is reduced to the symbol time-scale, instead of the physical clock.

As the only required feedback is the block average SINR, the underlying technique is referred to as open-loop MIMO. We only study here a simplif ed attachment mechanism relying on constant transmission powers. At a frst glance, this assumption may appear strongly suboptimal, but its main advantage relies on interference stabilisation. On the other hand, adapting a BS power to reduce interference on a given user has a limited effect due to the presence of numerous other interfering BSs. But the main advantage of this approach is the true distributed automatic adaptation and selection of resources based on local measurements only.

If it is easy in the literature to fnd works on multi-cell 
processing and clustering [12], [13], it is harder to fnd a practical criterion to decide on cooperation. Most of the time, a predef ned separation (based on distance or received signal strength) between inner and outer-users is considered, using Reuse1 or CoMP. Thus, the main goal of this paper is to provide the numerical evaluation, for three capacity metrics, of the potential gain of CoMP used in conjunction with the MIMO transmission.

Section II details the assumptions and the system modelling. Section III provides the CoMP selection procedure. Section IV presents the simulation results, followed by a conclusion.

\section{SySTEM MODEL}

In the following, we denote $(.)^{H}$ the Hermitian transpose, $\mathbf{I}_{n}$ an identity squared matrix of size $n$. We use bold lower case letters for vectors and bold upper case letters for matrices. By default, $\log$ refers to the binary logarithm. The present study focuses only on downlink and is based on a dense small cell scenario, which typically corresponds to urban conditions with a high density of base-stations to ensure coverage continuity and user requirements. We aim at optimising the association of users to base station(s), to maximise the per-BS spectral eff ciency.

\section{A. Assumptions}

The proposed approach relies on the following rules and assumptions:

1) The whole system is considered as a snapshot and the time dimension is therefore omitted.

2) OFDMA-based physical layer is assumes (similar to that used in Wimax and LTE-A) and the per-block behaviour is considered, with a common beacon period followed up by a set of time-frequency RBs.

3) Each mobile is associated with at least one, the highest receiver power BS, referred to as the primary BS.

4) No power control is applied, therefore the interference level at a mobile user is almost stable, averaged blockwise over the fading, which is assumed Rayleigh frequency $f$ at within a resource block.

5) The feedback channel allows each mobile to send a vector of average powers received from all neighbourhood BSs. The feedback data is not the exact transmit channel state (CSI-T), but a power level averaged over the whole frequency band.

6) We consider MIMO transmissions, with $N_{t}$ antennas at each BS and $N_{r}$ at each mobile.

\section{B. Multi-cell coordination principle}

The BSs distributed coordination for the CoMP algorithm holds in four steps: power sensing, CoMP capacity computation, CoMP mode selection and RB allocation. The f rst step is done by the mobiles while the three others are done by the primary BS.

1) Power sensing. Each mobile measures the average power received from each neighbour BS during the beacon period. It returns a powers vector $P=\left[P_{1}, \cdots, P_{N}\right]$ to its primary BS.
2) CoMP capacity computation. A primary BS computes from each received $P$, the spectral eff ciency resulting from different CoMP association. This procedure is detailed in next section.

3) Multiple BSs association. According the computed eff ciency, the primary BS can then request help from other BSs, on a mobile per mobile basis.

4) The RB are allocated from many BSs. For the the sake of conciseness, we do not develop the algorithmic aspect here, let us only mention, that it is supposed that all the BSs accept the demands for cooperation and can offer enough resources for it.

\section{CoMP with SISO links}

The combination of signals from two or more BSs allows users to improve their SINR level: the total interference power is decreased signif cantly by using the strongest interferer(s) as useful signal(s).

Considering the SISO case, at a given mobile and on a given channel, the received (complex) signal is:

$$
y=\sum_{n=1}^{N} h_{n} \sqrt{p_{n} g_{n}} x_{n}+z,
$$

where $n$ refers to transmitting antennas, considering $N$ total cells in the system. $z$ is the thermal noise, normal random variable with a $\sigma_{z}$ standard deviation. $h_{n} \sim \mathcal{C N}(0,1)$ are the fast fading random variables with Rayleigh distributed envelope; $x_{n}$ is the transmitted corresponding symbol; $p_{n}$ and $g_{n}$ are respectively the transmitted power and the channel gain which comprises the path-loss and shadowing effects from the BS $n$ to the mobile. Contrary to the fast-fading, the shadowing is considered as slowly varying with respect to the period of measurement reports from mobiles. We then suppose that $P_{n}=p_{n} g_{n}$, the average received power (over fast-fading) is known at base stations.

We note $\mathcal{N}$ the set of BSs that serve a given user in a coordinated manner, to create the MISO link, and $N_{\text {comp }}$ its cardinality. We can then write the received signal separating useful signal and interference as:

$$
y=\sum_{n \in \mathcal{N}} h_{n} \sqrt{P_{n}} x_{n}+\sum_{k \notin \mathcal{N}} h_{k} \sqrt{P_{k}} x_{k}+z
$$

We assume a perfect CSI known at receiver side and coherent detection, but CSI is not known at transmitter side and a spacetime code (such as Alamouti code for 2BSs) is used. The received SINR is then:

$$
\gamma(\mathcal{N})=\frac{\sum_{n \in \mathcal{N}}\left|h_{n}\right|^{2} P_{n}}{\sum_{k \notin \mathcal{N}}\left|h_{k}\right|^{2} P_{k}+\sigma_{z}^{2}},
$$

The user's SINR obviously increases when more transmitting antennas are used to perform CoMP, thanks to the contribution of (strong) interferers. Moreover, CoMP offers a macro-diversity due to distant locations of the base stations. The capacity obtained, as the SINR, is a random variable that depends on the topology and fading realisations. 


\section{CoMP with MIMO links}

Present standards already integrate not only one but several antennas at both receivers and transmitters. In downlink, the MIMO channel from a station $n$ to a given mobile can be written as: $\mathbf{H}_{n}=\sqrt{g_{n}} \mathbf{A}_{n} \in \mathbb{C}^{N_{r} \times N_{t}}$, comprising the pathloss $g_{n}$, and the Gaussian circular complex random matrix A. In the general MIMO case, the total received signal is:

$$
\mathbf{y}=\sqrt{\frac{p_{m}}{N_{t}}} \mathbf{H}_{n} \mathbf{x}_{n}+\sum_{k \neq m} \sqrt{\frac{p_{k}}{N_{t}}} \mathbf{H}_{k} \mathbf{x}_{k}+\mathbf{z}
$$

where $\mathbf{y}, \mathbf{x}_{n}, \mathbf{x}_{k}$ and $\mathbf{z}$ are complex vector of size $N_{r}$ that represent the total received signals, the transmitted signals from desired BS $m$ and from interfering stations $k \neq n$ and the noise, respectively. In our case, the matrices $\mathbf{H}$ are not known at transmitter side, so no particular precoding can be made and so $\mathbb{E}\left(\mathbf{x}_{k} \mathbf{x}_{k}^{H}\right)=\mathbf{I}_{N_{t}}$.

Assuming an ideal receiver, the mutual information of MIMO with interference channel is given by [14]:

$$
\mathcal{I}\left(\mathbf{x}_{n}, \mathbf{y}\right)=\log \operatorname{det}\left(\mathbf{I}_{N_{r}}+\frac{P_{n}}{N_{t}} \mathbf{A}_{n} \mathbf{A}_{n}^{H} \mathbf{R}^{-1}\right),
$$

where $\mathbf{I}_{N_{r}}$ is a unitary diagonal matrix of size $N_{r} \times N_{r}$ and $\mathbf{R}=\sum_{k \neq n} \frac{P_{k}}{N_{t}} \mathbf{A}_{k} \mathbf{A}_{k}^{H}+\sigma_{z}^{2} \mathbf{I}_{N_{r}}$ is referring to the interference and noise.

Now, looking at CoMP with MIMO, we associate antennas from few neighbouring BS to perform a (virtual) MIMO. Fig.1 illustrates the scenario: a group of $N_{\text {comp }}$ BS transmitting with their $N_{t}$ antennas to a mobile, while stations that don't belong to $\mathcal{N}$ interfere. The received signal can be written as:

$$
\mathbf{y}=\mathbf{G}_{n} \mathbf{x}_{n}+\sum_{k \notin \mathcal{N}} \mathbf{H}_{k} \mathbf{x}_{k}+\mathbf{z}
$$

where $\mathbf{x}_{n} \in \mathbb{C}^{N_{t} N_{\text {comp }}}$ is the transmitted vector symbol, distributed on all serving antennas, and $\mathbf{G}_{n} \in \mathbb{C}^{N_{r} \times N_{t} N_{\text {comp }}}$, indexed by $n$, is the matrix that horizontally concatenates the matrices $\sqrt{\frac{p_{n}}{N_{t}}} \mathbf{H}_{n}, \forall n \in \mathcal{N}$. $\mathbf{G}_{s}$ is similar to a $N_{r} \times N_{t} N_{\text {comp }}$ MIMO channel with non identically distributed random variables, since the $p_{n}$ values are specif c for each $n$. We can derive the mutual information as[14][15, 10.3]:

$$
\begin{aligned}
\mathcal{I}_{\mathcal{N}}\left(\mathbf{x}_{n}, \mathbf{y}\right) & =\log \operatorname{det}\left(\mathbf{I}_{N_{r}}+\mathbf{G}_{n} \mathbf{G}_{n}^{H} \mathbf{R}^{-1}\right) \\
& =\sum_{r=1}^{\operatorname{Rank}} \log \left(1+\lambda_{r}(\mathcal{N})\right),
\end{aligned}
$$

with $\mathbf{R}=\sum_{k \notin \mathcal{N}} \frac{P_{k}}{N_{t}} \mathbf{A}_{k} \mathbf{A}_{k}^{H}+\sigma_{z}^{2} \mathbf{I}_{N_{r}}$ and with $\lambda_{r}$ and Rank $\leq \min \left(N_{r}, N_{t} N_{\text {comp }}\right)$ being the eigenvalues of $\boldsymbol{\Gamma}(\mathcal{N})=\mathbf{G}_{n} \mathbf{G}_{n}^{H} \mathbf{R}^{-1}$ and its rank, respectively. Eq.(3) is a particular case of $\boldsymbol{\Gamma}$ for SISO links.

\section{E. Outage probability}

The probability for a channel of a given user with a set $\mathcal{N}$ of cooperating stations to be in capacity outage (ie. that fails

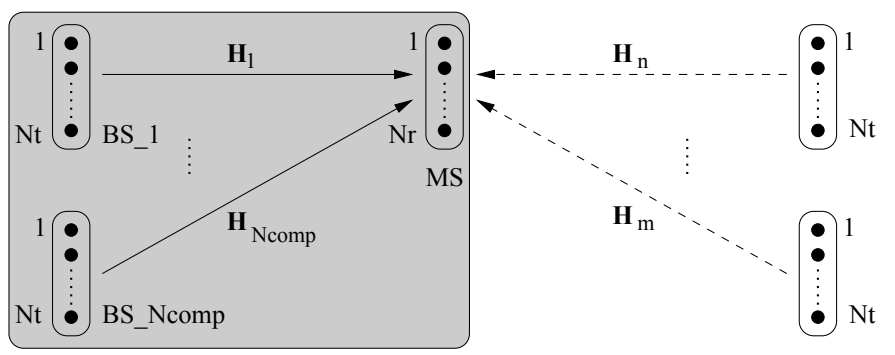

Fig. 1: MIMO cooperative transmission by $N_{\text {comp }}$ BS with $N_{t}$ antennas to the mobile with $N_{r}$ antennas, together with the co-channel interference from non-cooperating BSs.

to reach a target capacity $C_{t h}$ ) is, for the SISO case [16]:

$$
\begin{aligned}
& P_{\mathcal{N}}^{\text {out }}\left(C_{t h}\right)=\mathbb{P}\left(\log (1+\gamma(\mathcal{N}))<C_{t h}\right)= \\
& 1-\sum_{n \in \mathcal{N}}\left(e^{-\frac{\gamma_{t h} \sigma_{z}^{2}}{P_{n}}} \prod_{j \in \mathcal{N}, j \neq n} \frac{P_{n}}{P_{n}-P_{j}} \prod_{k \notin \mathcal{N}} \frac{P_{n}}{P_{k} \gamma_{t h}+P_{n}}\right) .
\end{aligned}
$$

For the MIMO case,

$$
\begin{aligned}
P_{\mathcal{N}}^{\text {out }}\left(C_{t h}\right) & =\mathbb{P}\left(\log \operatorname{det}(1+\boldsymbol{\Gamma}(\mathcal{N}))<C_{t h}\right) \\
& =\mathbb{P}\left(\sum_{r=1}^{\text {Rank }} \log \left(1+\lambda_{r}\right)<C_{t h}\right)
\end{aligned}
$$

has no tractable distribution, to the best of authors knowledge. However, one can more easily look at numerical distribution, depending on each received power value. Let $f_{\lambda_{r}}$ be the empirical PDF of $\log \left(1+\lambda_{r}\right)$, for $1<r<$ Rank, with respect to fading. Then, one can deduce the outage probability:

$$
P_{\mathcal{N}}^{\text {out }}\left(C_{t h}\right)=\int_{x=0}^{C_{t h}} f_{\lambda_{1}} * \cdots * f_{\lambda_{\text {Rank }}}(x) d x
$$

\section{Selection Of COOPERATING STATIONS}

Since only the average channel gain over the block can be available for known fading distribution, but not the instantaneous channel state, one of the possible system metrics is the capacity with outage $[15,4.2 .3]$. The goodput $C(\mathrm{bps} / \mathrm{Hz})$, is def ned as the user spectral eff ciency resulting from successful transmissions.

$$
C_{\text {out }}(\mathcal{N})=c\left(1-P_{\mathcal{N}}^{\text {out }}(c)\right),
$$

where $c=\log _{2}(1+\gamma(N))$ is the transmission data rate. Typically, for any acceptable outage probability (e.g. $1 \%, 10 \% \ldots$...) the corresponding transmission rate is deduced.

If the outage is not the criterion for a given user, once the set $\mathcal{N}$ is chosen, his goodput can be maximised with respect to the transmission rate $c$.

$$
C_{\text {opt }}(\mathcal{N})=\max _{c} c\left(1-P_{\mathcal{N}}^{\text {out }}(c)\right),
$$

Another possibility, linked to the information theory, is to evaluate the expectation of the mutual information, also referred to as Shannon, or ergodic capacity:

$$
C_{\text {exp }}(\mathcal{N})=\mathbb{E}(\log \operatorname{det}(1+\boldsymbol{\Gamma}(\mathcal{N})))
$$


To fairly compare the performance of mobiles attached to one or several BSs, we need to take into account the cooperation cost, or the cost of resources due to it. Consider a cellular network in which the mobiles can cooperate with two BS. If we decide to attach the mobile to two BSs instead of one, the resource consumption for this mobile is doubled (one for each cooperating station). Thus, a criterion for a mobile to select two BSs is the fact to have the goodput with CoMP at least twice better than without it. That way, we minimise the total amount of resource required by a mobile. This can be generalised for any number into the following utility function, used for example in [13], [17] and maximising a per-BS spectral eff ciency $S(\mathrm{bps} / \mathrm{Hz} / \mathrm{BS})$ :

$$
S(\mathcal{N})=\frac{C(\mathcal{N})}{N_{\text {comp }}},
$$

where $C$ is the capacity metric used, and could be either $C_{\text {out }}, C_{\text {opt }}$ or $C_{\text {exp }}$. To optimise network resources consumption, we then need to maximise $S(\mathcal{N})$ on $\mathcal{N}$, for each user. The next section provides numerical results for the selection of the optimal $N_{\text {comp }}$ value.

\section{NUMERICAL RESULTS}

To illustrate the interest of MIMO-CoMP, we present in this section the study of the probability for a mobile to select a given number $N_{\text {comp }}$ of cooperating BSs to optimise its own spectral eff ciency.

The target system is supposed to use OFDMA, where the entire bandwidth is divided into narrowband blocks to form time-frequency resource slots (e.g. in LTE, a Resource Block (RB) corresponds to a $200 \mathrm{kHz}$ bandwidth). We focus here on a per-block study, and so the outage probability and capacity formulations provided in previous sections, which are valid for a narrowband analysis, still hold.

The simulation setup is as follows. The BS are uniformly distributed on a 2D-plan, the propagation model is that of 3GPP MIMO Line-Of-Sight [18], with parameters' values resumed in Table I. According to this model, we simulate the received power levels at the mobiles. Up to $N_{\text {comp }}=8$ cooperating BSs are considered here for practical reasons.

Figure 2 shows the fraction of users that choose $N_{\text {comp }}$ BS to cooperate in order to maximise (15). Four different cases are presented here, referring to the following metrics: Fig.2a uses (12) with a fxed outage $P_{\mathcal{N}}^{\text {out }}(R)=1 \%$; Fig.2b uses (12) with a fxed outage $P_{\mathcal{N}}^{\text {out }}(R)=10 \%$; Fig.2c uses (13); and f nally Fig.2d uses (14) (note that y-axis scale differs in the last sub-f gure).

The impact of the outage requirement on the CoMP selection can be easily observed from the frst two f gures. The general tendency is that for a very low capacity outage probability (which can be required for example by a real-time application), the fraction of users that makes use of the CoMP is reduced. This is natural, since the mobiles tend to take the full advantage of the macro-diversity, in order to reduce the interference and eventually to create a link with a higher matrix rank. Nonetheless, both for $1 \%$ and $10 \%$ capacity outage it is

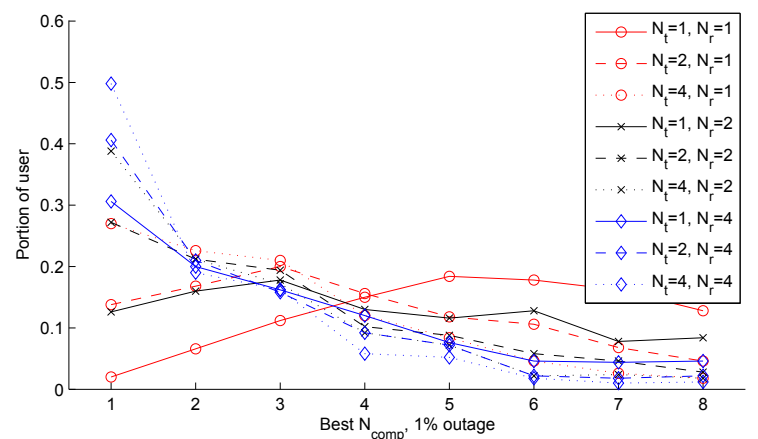

(a)

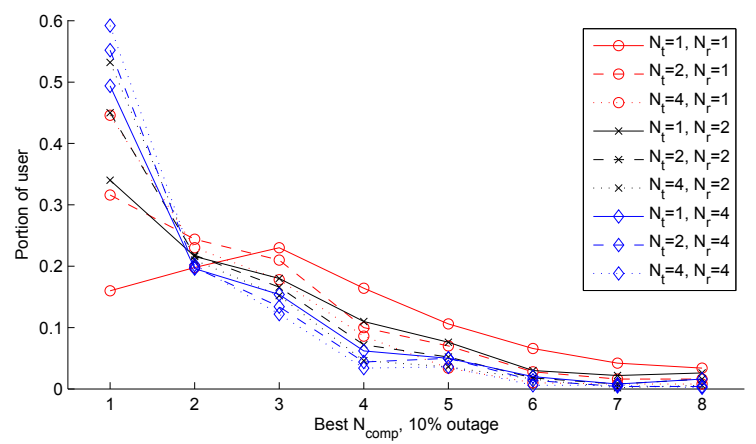

(b)

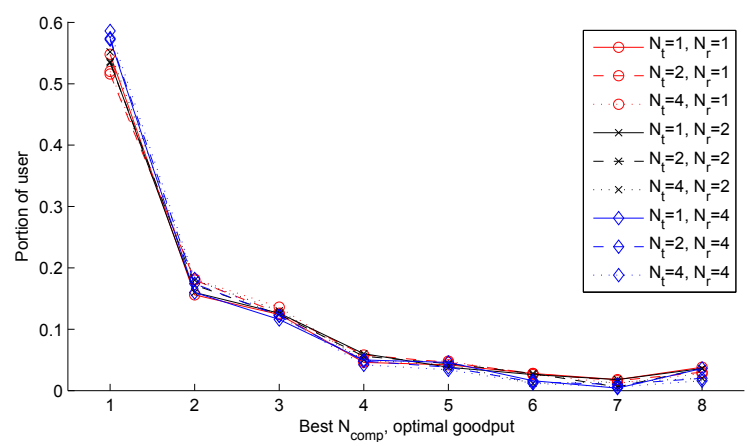

(c)

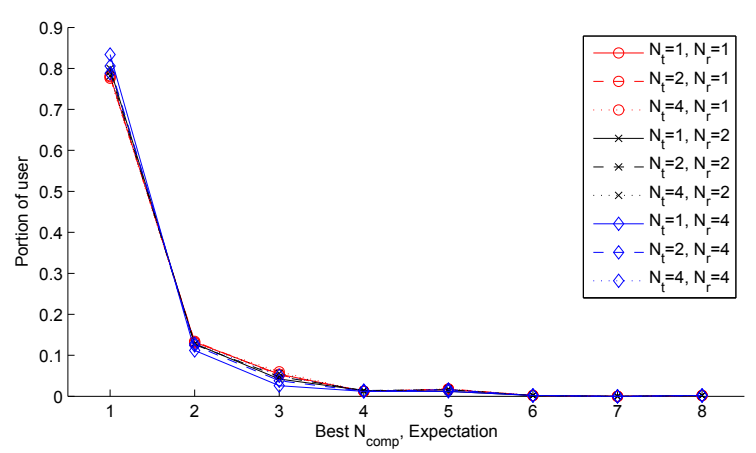

(d)

Fig. 2: Selection of the optimal $N_{\text {comp }}$ depending on the $N_{t} \times$ $N_{r}$ MIMO scheme used and the desired outage. (a) Capacity outage: $1 \%$ (b) Capacity outage: $10 \%$, (c) Optimised goodput, (d) Expectation. 


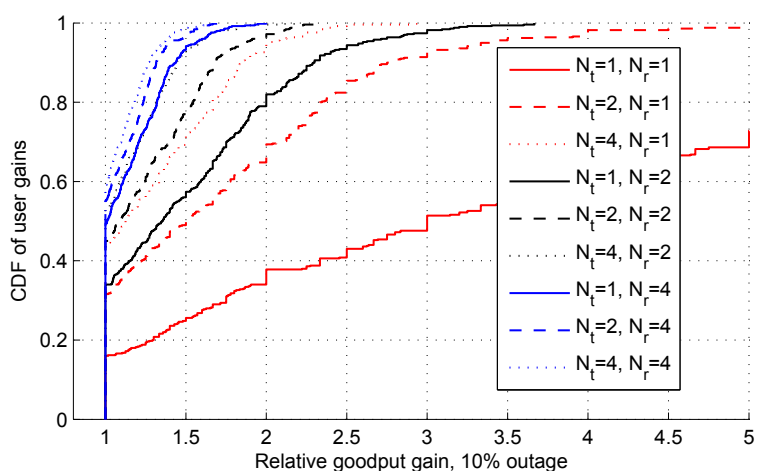

Fig. 3: CDF of the users' relative gain in spectral eff ciency between CoMP and no-CoMP. Outage requirement: $10 \%$.

interesting to notice that even for $(4 \times 4)$ MIMO only $50 \%$ and $60 \%$ of users will not select the CoMP, while for the others the CoMP still can be useful to increase the spectral eff ciency.

If the outage is not taken into account (which can be used, for instance, to maximise the total throughput), the CoMP is less employed by mobile, since some diversity gain is already taken into account by exploiting the whole distribution of the capacity. However, $40 \%$ to $50 \%$ of mobiles using the optimised goodput method still prefer to be attached to more than one BS. This percentage falls to about $20 \%$ when looking at expectation, but this metric imposes the transmitter to instantaneously adapt its transmission rate to the fading realisation, which is not feasible in practice. In the last two cases, it is interesting to note that the number of transmitting and receiving antennas has a very low impact on the CoMP choice. Obviously, this does not mean that capacities using different antennas numbers is the same or that the gain using CoMP is similar, but rather that the same fraction of users will still gain by using a CoMP.

Figure 3 illustrates spectral eff ciency gain of the CoMP. It shows the CDF of users' relative gain in spectral eff ciency, dividing (15) using the optimal $\mathcal{N}$ by (15) with $\operatorname{card}(\mathcal{N})=1$, when a $10 \%$ outage probability is required. For instance, about $60 \%$ of SISO communications double their spectral eff ciency with CoMP, while about $20 \%$ of $2 \times 2$ MIMO communications have a $50 \%$ gain. One can see no user will decrease its capacity using CoMP since the mobile can choose a single serving BS if needed. Following the previous remarks, we show here that the gain provided by CoMP is more signif cant when few antennas are set at mobiles and BSs.

\begin{tabular}{|c|c|}
\hline Parameter & Value \\
\hline BS density & $100 \mathrm{BS} / \mathrm{km}^{2}$ \\
RB Transmit power & $10 \mathrm{dBm}$ \\
RB Bandwidth & $200 \mathrm{kHz}$ \\
Thermal noise density & $-174 \mathrm{dBm} / \mathrm{Hz}$ \\
Path-loss (dB) [18], $d$ in m. & $-30.18-26 \log _{10}(d)$ \\
Shadowing standard dev. & $4 \mathrm{~dB}$ \\
Max $\left(N_{r}, N_{t}, N_{\text {comp }}\right)$ & $(4,4,8)$ \\
\hline
\end{tabular}

TABLE I: Simulation parameters.

\section{CONClusion}

Since frequency planning or manual conf guration is hardly practical in small cells, interference management through inter-cell coordination is one of the promising key features of future wireless standards. This paper analysed an openloop CoMP with MIMO Rayleigh channels and showed its utility by pointing out the probability for mobiles to improve their spectral eff ciency by selecting several BS. To satisfy low outage constraints, more mobiles require a multiple BS attachment to benef ts from lower interference and higher diversity.

\section{ACKNOWLEDGEMENT}

This work has been carried out in the frame of the joint lab between INRIA and Alcatel-Lucent Bell Labs on "Self Organizing Networks".

\section{REFERENCES}

[1] S. Jing, D. N. C. Tse, J. B. Soriaga, J. Hou, J. E. Smee, and R. Padovani. Multicell downlink capacity with coordinated processing. EURASIP Journal on Wireless Comm. Networks, 2008(5):1-19, 2008.

[2] Antti Toskala. "LTE advanced in 3GPP Release 10", keynote speach, COST2100, 9th MCM, Vienna, Austria, September 2009.

[3] M. C. Necker. Towards frequency reuse 1 cellular FDM/TDM systems. In MSWiM '06, pages 338-346, New York, USA, 2006. ACM.

[4] A. Alsawah and I. Fijalkow. Optimal frequency-reuse partitioning for ubiquitous coverage in cellular systems. In 15th European Signal Processing Conference (EUSIPCO), 2008.

[5] E. Bjornson, R. Zakhour, D. Gesbert, and B. Ottersten. Distributed multicell and multiantenna precoding: Characterization and performance evaluation. In IEEE GLOBECOM 2009, pages 1-6, nov. 2009.

[6] J. Hoydis, M. Kobayashi, and M Debbah. On the optimal number of cooperative base stations in network MIMO systems. 2010. Available online: http://arxiv.org/abs/1003.0332.

[7] M. C. Necker. Local interference coordination in cellular ofdma networks. Vehicular Technology Conference, 2007. VTC-2007 Fall. 2007 IEEE 66th, pages 1741-1746, 30 2007-Oct. 32007.

[8] A. L. Stolyar and H. Viswanathan. Self-organizing dynamic fractional frequency reuse in ofdma systems. In Proc. INFOCOM 2008. The 27th Conf. Computer Communications. IEEE, pages 691-699, 2008.

[9] 3GPP TR 36.902. Rel-9. E-UTRAN. Self-conf guring and selfoptimizing network (SON) use cases and solutions., Dec. 2009.

[10] Saad G. Kiani, Geir E. Øien, and David Gesbert. Maximizing multicell capacity using distributed power allocation and scheduling. In $W C N C$, 2007.

[11] O. Simeone, O. Somekh, H. V. Poor, and S. Shamai. Downlink multicell processing with limited-backhaul capacity. EURASIP Journal on Advanced Signal Process, 2009:1-10, 2009.

[12] A. Papadogiannis, D. Gesbert, and E. Hardouin. A dynamic clustering approach in wireless networks with Multi-Cell cooperative processing. In IEEE International Conference on Communications ICC'08, pages 4033-4037, Beijing, China, May 2008.

[13] J. Zhang, R. Chen, J.G. Andrews, A. Ghosh, and R.W. Heath. Networked MIMO with clustered linear precoding. IEEE Trans. on Wireless Communications, 8(4):1910-1921, 2009.

[14] R. S. Blum. Mimo capacity with interference. IEEE Journal on Selected Areas in Communications, 21(5):793-801, 2003.

[15] A. Goldsmith. Wireless Communications. Cambridge University Press (CUP), U.K., 2005.

[16] V. Garcia, J.-M. Gorce, and N. Lebedev. Outage Probability for MultiCell Processing under Rayleigh Fading. Accepted, IEEE Communications Letters, available online: http://hal.archives-ouvertes.fr/inria00519390/en/.

[17] V. Garcia, N. Lebedev, and J-M. Gorce. Multi-cell processing for uniform capacity improvement in full spectral reuse system. In COGnitive systems with Interactive Sensors, Paris, France, Nov. 2009. SEE, IET.

[18] 3GPP TR 25.996. Rel-9. Spatial channel model for MIMO simulations, Dec. 2009. 